\title{
Use of Chlorhexidine to Eradicate Oropharyngeal SARS- CoV-2 in COVID-19 Patients
}

\author{
Huang $\mathrm{YH}^{1}$ and Huang $\mathrm{JT}^{2 *}$ \\ ${ }^{1}$ University of Southern California, Keck School of \\ Medicine, USA \\ ${ }^{2}$ Division of Infectious Diseases, Good Samaritan \\ Hospital, USA \\ *Corresponding author: J ong T Huang, Division \\ of Infectious Diseases, Good Samaritan Hospital, Los \\ Angeles, California, USA
}

Received: February 13, 2021; Accepted: March 19, 2021; Published: March 26, 2021

\begin{abstract}
Objective: As public distribution of a vaccine against severe acute respiratory syndrome coronavirus 2 (SARS-CoV-2) is in underway, prevention of coronavirus disease 2019 (COVID-19) relies on minimizing spread. In this study, chlorhexidine gluconate was investigated as a topical antimicrobial agent against SARS-CoV-2.

Methods: This was a randomized, prospective cohort study using chlorhexidine as an oral rinse and posterior oropharyngeal spray in hospitalized COVID-19 patients. The primary outcome was presence or absence of laboratory-confirmed SARS-CoV-2 in the oral and oropharyngeal cavities after four days of chlorhexidine use and standard of care (study group) or standard of care only (control group).

Results: SARS-CoV-2 was eliminated from the oropharynx in $62.1 \%$ of patients who used chlorhexidine as an oral rinse, versus $5.5 \%$ of the control group patients. In patients who used a combination of oral rinse and oropharyngeal spray, $86.0 \%$ eliminated oropharyngeal SARS-CoV-2, versus $6.3 \%$ of control patients.

Conclusions: Chlorhexidine is a simple and safe addition to current COVID-19 prevention guidelines and may play a significant role in reducing disease spread.

Keywords: SARS-CoV-2; COVID-19; Disinfectants
\end{abstract}

\section{Background}

Since the emergence of the severe acute respiratory syndrome coronavirus 2 (SARS-CoV-2) in December 2019, there have been over 105.4 million confirmed cases of coronavirus disease 2019 (COVID-19) worldwide resulting in over 2.3 million deaths [1,2]. In the absence of effective systemic antimicrobial agents, prophylaxis is crucial for disease control. The gold standard of epidemic disease prophylaxis is a combination of vaccine immunization, use of prophylactic antimicrobial agents, and isolation from the causal microorganism. As public distribution of a SARS-CoV-2 vaccine is in underway, prophylactic recommendations currently focus on isolation from the virus via social distancing, mask wearing, hand washing, and disease tracing [3]. There is emerging evidence that topical antimicrobial agents may also be useful in preventing the disease spread [4,5]. In this study, the authors investigated the use of chlorhexidine gluconate as an oropharyngeal antimicrobial agent against SARS-CoV-2 infection.

\section{Materials and Methods}

This was a prospective cohort study performed at four community hospitals in Los Angeles, CA. All patients were consented prior to their participation. Patients with COVID-19 infection between May 20, 2020, and December 15, 2020 who were admitted to the COVID-19 wards were identified. All COVID-19 diagnoses were confirmed via detection of SARS-CoV-2 in nasopharyngeal swab specimens by real-time reverse Transcription Polymerase Chain
Reaction (rRT-PCR). The study population included patients who were able to follow instructions to use chlorhexidine as an oral rinse. Patients with nasogastric or endotracheal tubes placed were excluded from the study. Patients who were symptomatic for over a week prior to admission were also excluded.

From this population, patients were randomly assigned to the study and control groups. Both groups received the standard of care in their treatment plans. The study group was given chlorhexidine to use as an oropharyngeal rinse while the control group was not. For each administration of chlorhexidine, a unit dose cup containing $1 / 2$ ounce (15 milliliters) of commercially available chlorhexidine gluconate $(0.12 \%)$ was provided to each patient. Patients were then observed to self-administer the solution as a thorough oral rinse for 30 seconds twice a day. After 4 days, the oropharynx was swabbed and tested for the presence of SARS-CoV-2 by rRT-PCR.

Since an oral rinse alone would not reach the posterior oropharynx effectively, a chlorhexidine spray was added to the oral rinse regimen in the second study group. After the patient used chlorhexidine as an oral rinse as stated above, a provider used a spray applicator to deliver 3 sprays (a total of approximately 1.5 milliliters) of the chlorhexidine solution to the posterior oropharynx. To open the posterior pharynx, the patient was instructed to vocalize "ah" for 5 seconds while the solution was sprayed. This process was performed twice a day for 4 days. After four days of chlorhexidine administration, the oropharynx was swabbed and tested for presence of SARS-CoV-2 by rRT-PCR. A paired T-test was used to compare findings. 


\section{Results}

684 patients with positive SARS-CoV-2 infection were identified. The average onset of symptoms was 2-6 days prior to admission. Among the total study population, 294 patients were included in analysis, with 159 patients receiving chlorhexidine and 135 patients in the control group.

\section{Oropharyngeal specimens after oral rinse only}

A total of 121 patients were included in the analysis of using chlorhexidine as an oral rinse in COVID-19 patients (Table 1). Of the 66 patients in the chlorhexidine-treated group, 41 (62.1\%) were found to be negative and 25 (37.9\%) were found to be positive for SARS-CoV-2 in the oropharynx. On the same testing in the control group of 55 patients, 3 (5.5\%) tested negative and 52 (94.6\%) tested positive. The differences in the results were statistically significant between the two groups $(\mathrm{P}<0.01)$.

Patients in the study group used a chlorhexidine oral rinse twice a day for 4 days. Patients in the control group received no chlorhexidine. Oropharyngeal specimens tested for presence of SARS-CoV-2 by rRT-PCR on the fourth day. P-value of chlorhexidine-treated vs. control groups was $<0.01$.

\section{Oropharyngeal specimens after oral rinse with oropharyngeal spray}

Oropharyngeal specimens from 173 patients were included in the evaluation of using a chlorhexidine oral rinse with additional posterior oropharyngeal chlorhexidine spray in COVID-19 patients (Table 2). 80 of $93(86.0 \%)$ of the chlorhexidine-treated patients were negative for presence of SARS-CoV-2, while 5 of 80 (6.2\%) of the control patients were found to be negative for the virus. The differences in the results were statistically significant between the two groups $(\mathrm{P}<0.01)$.

Patients in the study group used a chlorhexidine oral rinse and posterior oropharyngeal spray twice a day for 4 days. Patients in the control group received no chlorhexidine. Oropharyngeal specimens tested for presence of SARS-CoV-2 by rRT-PCR on the fourth day. $\mathrm{P}$-value of chlorhexidine-treated $v s$. control groups was $<0.01$.

\section{Use of chlorhexidine in healthcare workers}

14 nurses and one physician in the hospitals where this study was conducted followed the above chlorhexidine oral rinse with oropharyngeal spray regimen twice a day in addition to social distancing, mask wearing, and hand washing to prevent contracting COVID-19. In this group of 15 healthcare workers, none were observed to develop SARS-CoV-2 infection during the course of this study. In contrast, the rate of COVID-19 among all healthcare workers in the hospitals during the same time period was near $50 \%$.

No adverse effects of using chlorhexidine as outlined were observed. Due to a shortage of SARS-CoV-2 testing kits, individual test results were not duplicated.

\section{Discussion}

Chlorhexidine is an antimicrobial agent that is commonly used in the pre-operative preparation of skin to prevent post-operative infections, dental plaque prevention, and the prevention of ventilatorassociated pneumonia [6-8]. Due to its cationic nature, chlorhexidine
Table 1: Detection of SARS-CoV-2 by rRT-PCR in the oropharynx of COVID-19 patients after a four-day course of chlorhexidine oropharyngeal rinse.

\begin{tabular}{|c|c|c|c|}
\hline & Positive & Negative & Total \\
\hline Chlorhexidine-treated group & $25(37.9 \%)$ & $41(62.1 \%)$ & 66 \\
\hline Control group & $52(94.5 \%)$ & $3(5.5 \%)$ & 55 \\
\hline
\end{tabular}

Table 2: Detection of SARS-CoV-2 by rRT-PCR in the oropharynx of COVID-19 patients after a four-day course of chlorhexidine oropharyngeal rinse and posterior oropharyngeal spray.

\begin{tabular}{|c|c|c|c|}
\hline & Positive & Negative & Total \\
\hline Chlorhexidine-treated group & $13(14.0 \%)$ & $80(86.0 \%)$ & 93 \\
\hline Control group & $75(93.8 \%)$ & $5(6.2 \%)$ & 80 \\
\hline
\end{tabular}

has been shown to be effective in killing enveloped viruses [7]. As SARS-CoV-2 is an enveloped virus, chlorhexidine has been shown to be effective against SARS-CoV-2 [9].

In an effort to deliver chlorhexidine to the posterior oropharynx more effectively, a spray applicator was used to apply chlorhexidine to the posterior oropharynx directly. In this study population, the addition of the posterior oropharyngeal chlorhexidine spray was associated with elimination of SARS-CoV-2 from the oropharynx in $86.0 \%$ of patients, compared with $62.1 \%$ in patients who used chlorhexidine as an oral rinse alone. This result suggests that the applying chlorhexidine to the posterior oropharynx greatly improved clearance of SARS-CoV-2 from the oropharynx.

Chlorhexidine was noted to be very effective in preventing SARS-CoV-2 infection in a small group of healthcare workers when compared with the general hospital healthcare worker population. The observation is encouraging, but further study with a larger cohort is needed to draw further conclusions.

The data from this study show a significant elimination of SARS-CoV-2 with chlorhexidine application in the oral cavity and pharynx. The authors propose that the use of chlorhexidine as an oropharyngeal rinse may serve two purposes: 1) to prevent viral spread from COVID-19 patients to others and 2) to prevent SARSCoV-2 infection in the case of exposure to the virus.

For prevention of viral spread from SARS-CoV-2-infected individuals to others, the authors recommend using chlorhexidine gluconate $0.12 \%$ mouthwash twice a day as follows: 1 ) spray 1 milliliter to the nares, 2) rinse the throat thoroughly with 15 milliliters for at least 30 seconds, and 3) use a spray applicator to spray the posterior throat 3 times ( 1.5 milliliters). This process may be continued until the virus is naturally cleared from the body, which takes approximately 2-3 weeks [10]. For post-exposure prophylaxis, the authors recommend the above chlorhexidine regimen for 2-4 days.

The authors must state clearly that chlorhexidine cannot be used to treat COVID-19, and strongly caution against ingesting chlorhexidine in any attempt to eradicate the disease.

\section{Conclusion}

Chlorhexidine used as an oral rinse and posterior oropharyngeal spray is a simple and safe addition to the current COVID-19 prevention guidelines and may have significant effects on controlling the spread of the disease. When used with vaccination, proper social distancing, mask wearing, and hand washing, chlorhexidine may 
contribute to a more robust disease prevention regimen.

\section{Institutional Review Board Statement}

The study was conducted according to the guidelines of the Declaration of Helsinki and approved by the Ethics Committee of Avanti Hospitals (May 11, 2020).

\section{Informed Consent Statement}

Informed consent was obtained from all subjects involved in the study.

\section{References}

1. World Health Organization. Weekly epidemiological update-7 February 2021. 2021.

2. Khokhar M, Roy $D$, Purohit $P$, Goyal M, Setia P. Viricidal treatments for prevention of coronavirus infection. Pathog Glob Health. 2020; 114: 349-359.

3. CDC. Coronavirus disease 2019 (COVID-19): how to protect yourself and others. Atlanta, GA: US Department of Health and Human Services, CDC. 2020.

4. Carrouel F, Goncalves LS, Conte MP, Campus G, Fisher J, Fraticelli L, et al Antiviral Activity of Reagents in Mouth Rinses against SARS-CoV-2. J Dent Res. 2020; 100: 124-132.
5. Meyers C, Robison R, Milici J, Alam S, Quillen D, Goldenberg D, et al. Lowering the transmission and spread of human coronavirus. J Med Virol. 2021: 93: 1605-1612.

6. Anderson DJ, Podgorny K, Berrios-Torres SI, Bratzler DW, Dellinger EP, Greene L, et al. Strategies to prevent surgical site infections in acute care hospitals: 2014 update. Infect Control Hosp Epidemiol. 2014; 35: 605-627.

7. Jones CG. Chlorhexidine: is it still the gold standard? Periodontol 2000. 1997; 15: 55-62.

8. Houston S, Hougland P, Anderson JJ, LaRocco M, Kennedy V, Gentry LO. Effectiveness of $0.12 \%$ chlorhexidine gluconate oral rinse in reducing prevalence of nosocomial pneumonia in patients undergoing heart surgery. Am J Crit Care. 2002; 11: 567-570.

9. Yoon JG, Yoon J, Song JY, Yoon SY, Lim CS, Seong $\mathrm{H}$, et al. Clinical Significance of a High SARS-CoV-2 Viral Load in the Saliva. J Korean Med Sci. 2020; 35: e195.

10. Liu Y, Yan LM, Wan L, Xiang TX, Le A, Liu JM, et al. Viral dynamics in mild and severe cases of COVID-19. Lancet Infect Dis. 2020; 20: 656-657. 